\title{
La implementación de las TIC en el aula de música de Secundaria en comparación a metodologías tradicionales de enseñanza como la clase magistral. Estudio de caso.
}

\author{
Jesús Carrascosa Cambra ${ }^{\mathrm{a}}$, Conrado Carrascosa López \\ ${ }^{a}$ Universitat Valencia, Spain, chechucarras@gmail.com, ${ }^{b}$ Universitat Politècnica Valencia, Spain, \\ concarlo@upvnet.upv.es
}

\begin{abstract}
Resumen
Las Nuevas Tecnologías han facilitado la vida de las personas en muchos aspectos, y tienen una importancia capital en la sociedad de la información y el conocimiento actual. Sin embargo, el sistema educativo tiende a ser reacio a estos nuevos avances, decantándose por los métodos tradicionales de enseñanza. Este trabajo de investigación pretende comprobar si la implementación de las TIC como herramienta didáctica, en el aula de música de la ESO, conlleva una mejora en el aprendizaje significativo del alumnado con respecto a métodos tradicionales de enseñanza como la clase magistral. Para demostrarlo se coge una muestra de alumnos de la ESO de características similares que se divide en dos grupos, a los que se les explica la misma Unidad Didáctica de contenidos. A un grupo a través del uso de las TIC y al otro sin servirse de ellas. Como parte transversal del estudio, también se implementan dos metodologías docentes innovadoras como el aprendizaje ubicuo y el aprendizaje dialógico. Posteriormente, se pasa a los dos grupos la misma prueba de evaluación objetiva, a través de la cual se compara el nivel de asimilación de los contenidos de cada grupo. Los resultados muestran como en el grupo en que se emplearon las TIC, se produje una mejora notable en los resultados de la prueba respecto al grupo en que no se utilizaron las TIC.
\end{abstract}

Palabras clave: TIC, ESO, música, innovación educativa, metodologías innovadoras.

\section{Introducción}

A lo largo de las últimas décadas, los avances tecnológicos han facilitado y mejorado en muchos aspectos la calidad de vida de las personas. Sin embargo, la enseñanza reglada en 
La implementación de las TIC en el aula de música de Secundaria en comparación a metodologías tradicionales de enseñanza como la clase magistral. Estudio de caso.

nuestros centros educativos no ha evolucionado tanto. La práctica habitual, por lo general, es que el profesor base su explicación en el libro de texto, apoyándose en la pizarra, mientras los alumnos, en el mejor de los casos, escuchan y toman apuntes pasivamente. Esta metodología no es siempre la más eficaz.

El presente trabajo de investigación pretende aportar evidencias que demuestren que el uso de las nuevas tecnologías en el aula de música de Secundaria, a la vez que de metodologías docentes innovadoras como el aprendizaje dialógico y el aprendizaje ubicuo, suponen una mejora en la calidad de las explicaciones y por tanto de los resultados académicos del alumnado, en comparación a la clase magistral.

Con este propósito, se diseña el siguiente estudio de caso: coger una muestra de alumnos de la ESO de características similares y formar dos grupos. A los dos grupos se les explica la misma unidad didáctica de contenidos, a un grupo a través del uso de las TIC, y a otro sin servirse de ellas. A los dos grupos se les pasará la misma prueba de evaluación objetiva, con la que medir su nivel de asimilación de los contenidos de la explicación. Los resultados se compararán, y en base a ellos se elaborarán los resultados y conclusiones del estudio, comprobando si la utilización de las TIC en la práctica docente ha supuesto o no una mejora considerable en el aprendizaje significativo del alumnado.

\section{Marco teórico}

La creciente relevancia de las Tecnologías de la Información y la Comunicación (TIC) ha transformado a lo largo de las últimas décadas la vida de las personas (Gerick, Fickelmann y Bos, 2017), motivo por el cual el mundo no puede permanecer ajeno a las repercusiones del avance científico-tecnológico sobre la actividad humana (Martinez, 2015). Tampoco el sistema educativo.

Son numerosos los autores que han estudiado en profundidad las TIC como herramientas válidas y necesarias para ser implementadas en las aulas. Esteve, Navarro y Gómez (2019) afirman que la actual sociedad moderna impone el desafío de dejar a un lado todo tipo de recursos y métodos tradicionales para dar paso a las nuevas tecnologías y metodologías. En la misma línea, Waliño, Pardo, Peirats y San Martin (2018) analizan como la formación en competencia digital es uno de los grandes desafíos en el ámbito educativo de nuestro tiempo. Es necesario dar respuesta a un alumnado que ya ha nacido en la era digital. De no hacerlo así, la educación puede llegar a quedarse anclada y perder su operatividad (Martinez, 2015). 
Dentro del ámbito de la Educación Secundaria Obligatoria, la utilización de las TIC permite desarrollar competencias básicas diversas, tales como la competencia comunicativa, lingüística y audiovisual, la competencia en el tratamiento de la información y la competencia digital; la competencia de aprender a aprender y la competencia de autonomía e iniciativa personal (Martí y Garcia, 2014). En su tesis doctoral, Peña (2010) pone de manifiesto las aportaciones y beneficios que el trabajo con las TIC ofrece al alumnado. En la misma línea, Álvarez y Villodre (2019), diseñan una serie de rúbricas con las que evaluar la influencia de las TIC en el proceso de enseñanza/aprendizaje, con las que demuestran la influencia positiva de las TIC en el aprendizaje de contenidos.

En relación al impacto de las TIC en la educación musical en Secundaria, los autores Chao, Román y Chao (2017), llevan a cabo un interesante trabajo. Para medir el impacto de las TIC en el aula de música forman dos grupos distintos: uno de ellos trabaja a la "manera tradicional", y el otro a partir de distintas herramientas TIC. Más tarde, se miden objetivamente los resultados, y estos demuestran como el aprendizaje musical a través de las TIC mejora en el $100 \%$ de los parámetros analizados respecto de la metodología tradicional, poniendo de evidencia que la educación musical debería incorporar las nuevas tecnologías para facilitar el desarrollo de la pedagogía musical de las escuelas secundarias (Ferreira y Ricoy, 2017). Chao et al. (2017) llevan a cabo otro estudio de caso en el que comprueban como el uso de las TIC en educación musical mejora el aprendizaje y la integración de los estudiantes con discapacidades auditivas en la asignatura de música. Por otro lado, Hwang e Ilari (2019) analizan de manera magistral al alumnado que encontramos en nuestras aulas de música y la importancia que tiene para ellos el audiovisual, la multimodalidad y la tecnología, concluyendo que es necesario integrar estos elementos en nuestra enseñanza.

Por último, Martos (2016) destaca en su tesis doctoral como la figura del docente es trascendental para poder implantar y desarrollar adecuadamente las TIC en los contextos educativos. "El nuevo profesor de música debe hacer frente a una nueva realidad educativa, marcada por una auténtica revolución tecnológica, donde la formación del profesorado es clave para dar respuesta a esta" (p.100).

\section{Marco metodológico}

\subsection{Método y metodologías docentes empleadas}

Para la realización de este trabajo se emplea la metodología de investigación cuantitativa. Por otro lado, también se implementan tres metodologías docentes diferentes, la clase magistral, el aprendizaje dialógico y el aprendizaje ubicuo. 
La implementación de las TIC en el aula de música de Secundaria en comparación a metodologías tradicionales de enseñanza como la clase magistral. Estudio de caso.

La clase magistral es la más conocida de todas ellas, en la cual la explicación se centra fundamentalmente en la exposición verbal por parte del profesor de los contenidos sobre la materia objeto de estudio (Fortea, 2019). Por su parte, el aprendizaje dialógico defiende que la interacción entre ponente y oyentes es imprescindible para el aprendizaje. En esencia, se define como el que resulta de las interacciones que produce el diálogo igualitario en el que diferentes personas aportan argumentos en condiciones de igualdad, para llegar a un consenso común (Sandúa, González y López, 2014). Por último, el aprendizaje ubicuo es el que se produce en cualquier lugar y momento; la tecnología ubicua (informática cercana a la persona, por ejemplo, un móvil) potencia considerablemente este tipo de aprendizaje. La formación ubicua integra el aprendizaje y la tecnología ubicua dentro de una estrategia formativa y uno de sus frutos más conocidos es el m-learning (o Mobile Learning), que pretende aprovechar todas las posibilidades que los dispositivos móviles actuales ofrecen y aplicarlos a la práctica docente. En este sentido, Burbules (2012) defiende que el futuro de la formación docente tendrá que abordar el aprendizaje ubicuo: la posibilidad de acceder a la información en cualquier lugar o cualquier momento.

\subsection{Diseño de la investigación}

Para comprobar si la implementación de las TIC en el aula de música de la ESO conlleva una mejora en el aprendizaje significativo del alumnado, se diseña el siguiente estudio de caso. Se coge una muestra de alumnos de la ESO de características similares del IES Abastos de Valencia y se divide la muestra total en dos grupos distintos. A los dos grupos se les explica en una sesión de 55 minutos la misma unidad didáctica de contenidos, elaborada previamente y que trata la música en 8 dimensiones. A un grupo se le expone la unidad mediante el uso de las TIC, y al otro grupo no. Una vez finalizada la explicación, a los dos grupos se les pasa la misma prueba de evaluación objetiva tipo test. Los resultados se analizan y comparan, y en base a ellos se elaboran los resultados y conclusiones de este trabajo.

Muestra: mediante un muestreo probabilístico (aleatorio), se toman 4 clases del centro de los cursos de $2^{\circ}$ y $3^{\circ}$ de la ESO:

- El Grupo A (o Grupo Clase Magistral), está compuesto por las clases de $2^{\circ}$ ESO E y $3^{\circ} \mathrm{ESO} \mathrm{W}$.

- El Grupo B (o Grupo TIC), está compuesto por las clases de $2^{\circ}$ ESO V y $3^{\circ}$ ESOV.

Se procede así porque en las muestras aleatorias cada elemento de población tiene una probabilidad igual, o una probabilidad cuantificable, de ser seleccionado (Clark, 2002). La 
muestra total es de 97 alumnos: la suma total de estudiantes de las clases seleccionadas aleatoriamente, que acudieron al instituto el día en que se realizó el estudio de caso.

\section{Explicación de las sesiones:}

Con el Grupo A, se imparte una clase magistral, apoyando la explicación teórica únicamente con pizarra y tiza. No obstante, se incide en involucrar al máximo posible a los alumnos, tratando de dirigir la sesión a partir del feedback que estos vayan proporcionando (aprendizaje dialógico). Todo esto sin utilizar ninguna herramienta TIC.

Con el Grupo B, se explica exactamente la misma unidad didáctica de contenidos que con el Grupo A, pero con el apoyo de las TIC:

- Se pide a los alumnos que busquen con sus teléfonos móviles (m-learning) la canción: Dilbar, de Satyameva Jayate, una de las primeras canciones que se ha compuesto y producido originariamente para ser escuchada en 8 dimensiones. Se les da 5 minutos para que la escuchen con auriculares. Se pretende empezar la sesión con una experiencia vivencial de lo que supone la música en $8 \mathrm{D}$.

- A continuación se proyecta para toda la clase un video del Youtuber español Jaime Altozano, en el que explica la música en 8 Dimensiones. Adjunto el enlace: https://www.youtube.com/watch?v=e6Ekz7ZDV-w\&t=3s. La sesión se desarrolla a partir de este video, que tiene un gran interés, puesto que además de dar una explicación muy didáctica acerca del tema en cuestión, pone numerosos ejemplos y acompaña el video con gran cantidad de imágenes, esquemas y gráficos. Asimismo, en la segunda parte del video, Jaime Altozano produce música en 8 dimensiones, explicando paso a paso cómo hacerlo.

De igual manera que con el Grupo A, con el Grupo B también se implementa el aprendizaje dialógico, para tratar de involucrar lo máximo posible al alumnado. Y cuando es necesario, el profesor complementa la explicación.

\section{Resultados}

Los datos extraídos de la prueba de evaluación objetiva tipo test, corroboran la hipótesis de partida del estudio, que apuntaba a que la implementación de las TIC en el aula conlleva una mejora en los resultados y aprendizaje significativo del alumnado, con respecto a los métodos tradicionales de enseñanza. 
La implementación de las TIC en el aula de música de Secundaria en comparación a metodologías tradicionales de enseñanza como la clase magistral. Estudio de caso.

A continuación, se muestra la nota media obtenida en la prueba del Grupo A (Grupo clase magistral) en comparación al Grupo B (TIC). Estos son los resultados:

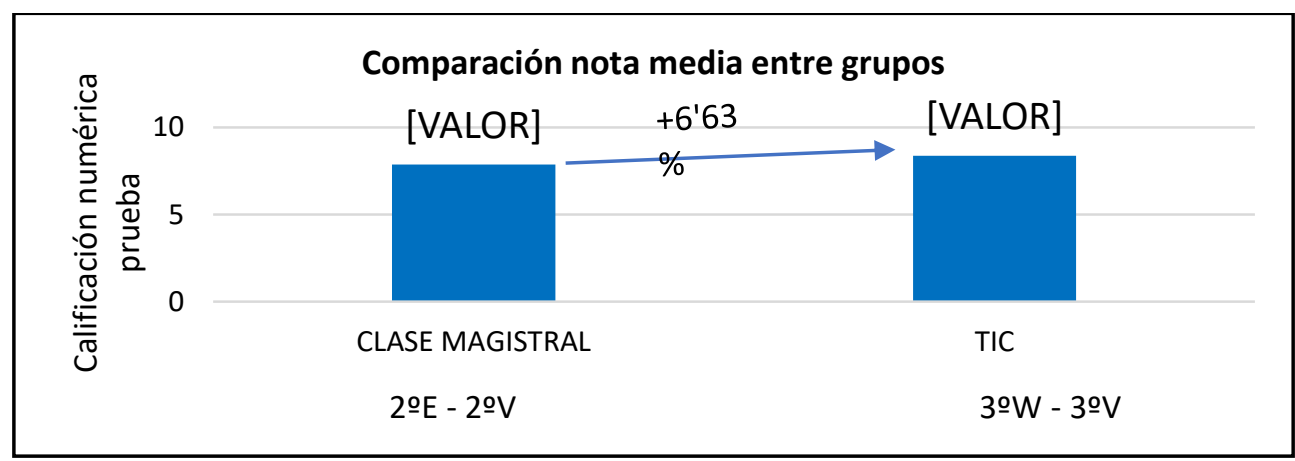

Fig. 1 Gráfica comparativa

Fuente: Elaboración propia

El grupo TIC obtuvo una calificación media 0`49 puntos mayor a la nota media del grupo clase magistral, lo que supone una mejora del 6'63\%.

Por otro lado, en el siguiente gráfico se muestra el porcentaje de alumnos que han respondido acertadamente cada pregunta, comparando el grupo clase magistral (Grupo A) con el grupo TIC (Grupo B). Estos son los resultados:

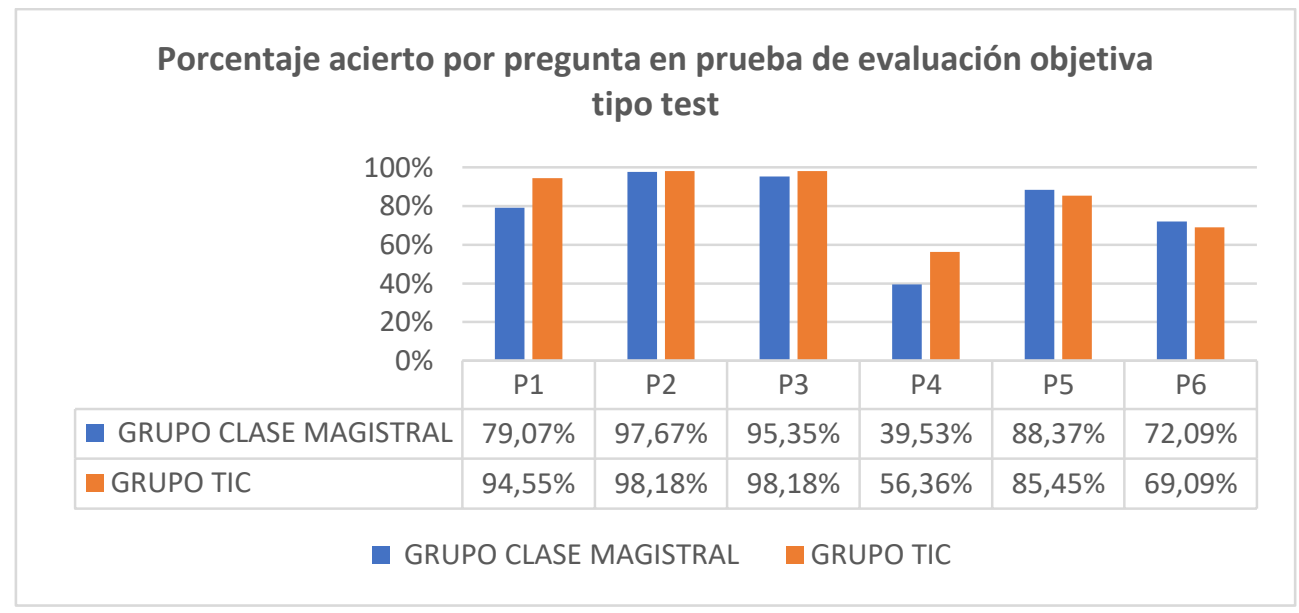

Fig. 2: Gráfica comparativa

Fuente: Elaboración propia

En las preguntas 2 y 3 , y 5 y 6 , los resultados son muy parejos entre los dos grupos. Sin embargo, en las preguntas 1 y 4 sí se observa una mejoría significativa en el Grupo B (Grupo TIC) respecto al Grupo A (Clase Magistral).

El Grupo B ha obtenido unos mejores resultados en la prueba que el Grupo A. 


\section{Conclusiones}

En vista de los resultados obtenidos, se concluye que el uso de las TIC como herramienta didáctica en el aula de música de la ESO, conlleva una mejora en el aprendizaje significativo del alumnado con respecto a la clase magistral.

En primer lugar, porque la implementación de las TIC como complemento a la labor docente facilita la conexión con el alumnado. En este estudio se propuso al grupo B seguir la explicación mediante un video de Youtube. A ojos de los alumnos, este hecho ha resultado ser mucho más atractivo que limitarse a escuchar al profesor y tomar los respectivos apuntes. Por otro lado, aplicar el m-learning al principio de la sesión con el grupo B, facilitó sobremanera conectar con el alumnado y consiguir que prestaran mayor atención a la exposición del docente.

Asimismo, se califica como muy satisfactorio el impacto creado por la metodología docente del aprendizaje dialógico. En las sesiones con ambos grupos se consiguió crear un ambiente de trabajo muy dinámico y participativo, en el que la mayoría de los alumnos aportaron sus conocimientos previos. La utilización de metodologías docentes que fomenten la participación e interacción activa del alumnado en la explicación, conllevan un mayor interés por su parte. Y a mayor interés del alumnado, mayor atención y retención de los conocimientos expuestos, lo que se traduce en unos mejores resultados académicos.

\section{Referencias}

Álvarez, B. T., y Villodre, M. D. M. B. (2019). Rúbricas para evaluar la influencia de las TIC en el proceso de enseñanza/aprendizaje: estudio de caso en Educación Secundaria. Aula de Encuentro, 21(1), 85-104. Extraído el 3 de Febrero de 2020 desde https://doi.org/10.17561/ae.v21i1.5

Burbules, N. C. (2012). El aprendizaje ubicuo y el futuro de la enseñanza. Encounters on education, 13. Extraído el 4 de Mayo de 2020 desde http://www.academia.edu/download/35719052/44728413-1-PB.pdf

Chao-Fernandez, R., Román-Garcíab, S., y Chao-Fernandeza, A. (2017). Analysis of the use of ICT through music interactive games as educational strategy. Procedia-Social and Behavioral Sciences, 237, 576-580. Extraído el 3 de Febrero de 2020 desde https://cyberleninka.org/article/n/734801.pdf

Chao-Fernandez, R., Román-García, S., y Chao-Fernandez, A. (2017). Online Interactive Storytelling as a strategy for learning music and for integrating pupils with hearing disorders into Early Childhood Education (ECE). Procedia-Social and Behavioral Sciences, 237, 17-22. Extraído el 3 de Febrero de 2020 desde https://cyberleninka.org/article/n/734290.pdf 
La implementación de las TIC en el aula de música de Secundaria en comparación a metodologías tradicionales de enseñanza como la clase magistral. Estudio de caso.

Clark-Carter, D. (2002), Investigación cuantitativa en psicología. Del diseño experimental al reporte de investigación, México, Oxford University Press.

Esteve, M. I. V., Navarro, A. V., y Gómez, S. L. (2019). Uso de materiales didácticos digitales en las aulas de Primaria. Campus Virtuales, 8(2), 103-119. Extraído el 5 de Febrero de 2020 desde http://www.uajournals.com/ojs/index.php/campusvirtuales/article/view/516

Ferreira, V., y Ricoy, M. C. (2017). Contribuição dos manuais de educação musical para a utilização das TIC. Cuadernos. info, (40). Extraído el 5 de Febrero de 2020 desde https://dialnet.unirioja.es/servlet/articulo?codigo $=6032877$

Fortea Bagán, M. Á. (2019). Metodologías didácticas para la enseñanza/aprendizaje de competencias. Materiales para la docencia universitaria de la Universitat Jaume I, $\mathrm{n}^{\mathrm{a}} 1$. Extraído el 5 de Febrero de 2020 desde http://dx.doi.org/10.6035/MDU1

Gerick, J., Eickelmann, B., y Bos, W. (2017). School-level predictors for the use of ICT in schools and students' CIL in international comparison. Large-scale Assessments in Education, 5(1), 5. Extraído el 3 de Febrero de 2020 desde https://doi.org/10.1186/s40536-017-0037-7

Hwang, Y., y Ilari, B. (2019). Music Teachers' Use of Online Video Platforms (OVPs) in Lesson Design and Instruction. Revista Latinoamericana de Tecnología Educativa-RELATEC, 18(2), 45 55. Extraído el 3 de Febrero de 2020 desde http://hdl.handle.net/10662/10436

Martí Climent, A., y Garcia Vidal, P. (2014). El treball per projectes amb les TIC a l'àrea de Llengua i Literatura. Una experiència didàctica en la formació del professorat de Secundària. Articles de Didàctica de la Llengua i la literatura, 64. Extraído el 5 de Febrero de 2020 desde http://hdl.handle.net/10550/66506

Martínez, J. A. C. (2015). El uso de las TIC, dispositivos móviles y redes sociales en un aula de la educación secundaria obligatoria. Tesis Doctoral, Universidad de Granada. Extraído el 3 de Febrero de 2020 desde https://digibug.ugr.es/handle/10481/42209

Martos Sánchez, E. (2016). El profesor de música de enseñanza secundaria ante el reto Andaluz de la escuela TIC 2.0. Tesis Doctoral, Universidad de Granada. Extraído el 3 de Febrero de 2020 desde https://digibug.ugr.es/handle/10481/42253

Peña Mecina, A. (2010). Enseñanza de la geometría con TIC en Educación Secundaria Obligatoria. Tesis doctoral, UNED. Extraído el 3 de Febrero de 2020 desde http://espacio.uned.es/fez/eserv/tesisuned:Educacion-Apena/Documento2.pdf

Sandúa, M. C., González, A. G., \& López, A. M. M. (2014). Aprendizaje dialógico y grupos interactivos en educación física. Retos: nuevas tendencias en educación física, deporte y recreación, (25), 174-179. Extraído el 4 de Mayo de 2020 desde https://dialnet.unirioja.es/servlet/articulo?codigo $=4555265$

Waliño Guerrero, M. J., Pardo Baldoví, M. I., Peirats Chacón, J., y San Martín Alonso, Á. (2018). Digital citizenship and the use of mobile devices in educational institutions. The European Conference on Educational Research (ECER). Extraído el 5 de Febrero de 2020 desde http://hdl.handle.net/10550/67780 